\title{
SPECTRAL THEORY OF GENERALIZED TOEPLITZ OPERATORS
}

BY

R. G. DOUGLAS AND CARL PEARCY

1. Introduction. In this note we consider a class of (bounded) operators on Hilbert space that properly contains the much-studied class of Toeplitz operators, and we show that virtually all of the results of [2] concerning spectral properties of Toeplitz operators are valid for operators in this larger class.

We devote $\$ 2$ to preliminary definitions and examples. The concept of a "Riesz system" is first introduced and then is used to define the class of generalized Toeplitz operators. Several examples of Riesz systems are set forth to illustrate the generality of the concept. In $\$ 3$ the spectral inclusion theorem for generalized Toeplitz operators is proved, i.e., it is shown that the spectrum of any given generalized Laurent operator is a subset of the spectrum of the associated generalized Toeplitz operator. This result is then used to recover several of the theorems of [2] for generalized Toeplitz operators. $\$ 4$ is devoted to questions concerning isomorphisms between Riesz systems and ways in which these systems can differ from the "classical" Riesz system that gives rise to Toeplitz operators. In $\$ 5$ we make some concluding remarks.

2. Generalized Toeplitz operators. Let $\mathscr{H}$ be any (not necessarily separable) infinite-dimensional Hilbert space, and let $\boldsymbol{R}$ be a maximal abelian von Neumann (v. N.) algebra of operators acting on $\mathscr{H}$. A (closed) subspace $\mathscr{K} \neq\{0\} \subset \mathscr{H}$ is said to be a weak Riesz subspace for $\boldsymbol{R}$ if each nonzero $f \in \mathscr{K}$ is a separating vector for $R$. By definition this means that if $T \in R$ and $T f=0$, then $T=0$. By virtue of a well-known theorem for $v$. N. algebras [3], this is equivalent to supposing that if $f$ is any nonzero vector in $\mathscr{K}$, then the linear manifold $R f$ is dense in $\mathscr{H}$. A proper subspace $\mathscr{K} \subset \mathscr{H}$ is a Riesz subspace for $R$ if both $\mathscr{K}$ and $\mathscr{K}^{\perp}$ are weak Riesz subspaces for $R$. A triple $(\mathscr{H}, R, \mathscr{K})$ is called a Riesz system if $\mathscr{H}$ is an infinite-dimensional Hilbert space, $R$ is a maximal abelian v. N. algebra on $\mathscr{H}$, and $\mathscr{K} \subset \mathscr{H}$ is a Riesz subspace for $\boldsymbol{R}$. We shall see below (Proposition 3.3) that this implies that both $\mathscr{K}$ and $\mathscr{K}^{\perp}$ are infinitedimensional. If $(\mathscr{H}, \boldsymbol{R}, \mathscr{K})$ is any Riesz system under consideration, the linear operator that projects $\mathscr{H}$ onto the subspace $\mathscr{K}$ will always be denoted by $P$. Furthermore, every operator $A \in \boldsymbol{R}$ will be called a gener-

Presented to the Society, January 27, 1965; received by the editors April 7, 1964. 
alized Laurent (GL) operator, and every operator of the form $P A$ acting on the Hilbert space $\mathscr{K}$ will be called a generalized Toeplitz (GT) operator. Thus every Riesz system gives rise to families of (GL) and (GT) operators, and it is appropriate to set forth some examples of Riesz systems:

EXAmpLe 1. Let $\mu$ be normalized Lebesgue measure on the unit circle $C$ in the complex plane, and let $\widetilde{\mathscr{E}}$ be the Hilbert space $L^{2}(\mu, C)$. Let $\widetilde{\boldsymbol{R}}$ be the $\mathrm{v}$. $\mathrm{N}$. algebra of all multiplications by bounded measurable functions on $L^{2}(\mu, C)$. Then $\widetilde{\boldsymbol{R}}$ is maximal abelian and is in fact isomorphic to $L^{\infty}(\mu, C)$ as a Banach algebra. Finally, consider the orthonormal basis $\left\{z^{n}:|z|=1,-\infty<n<\infty\right\}$ for $L^{2}(\mu, C)$, and let $\widetilde{\mathscr{K}}$ be the subspace of $\mathscr{E}$ consisting of all $f \in L^{2}(\mu, C)$ such that the sequence $\left\{c_{n}\right\}_{n=-\infty}^{\infty}$ of Fourier coefficients of $f$ with respect to the above orthonormal basis satisfies $c_{n}$ $=0$ for all $n<0$. The fact that $(\widetilde{\mathscr{H}}, \widetilde{R}, \widetilde{\mathscr{K}})$ is a Riesz system follows immediately from the F. and M. Riesz theorem [7]. And, of course, the operators $A \in \widetilde{\boldsymbol{R}}$ are the "classical" Laurent operators, and the operators $P A$ on $\widetilde{\mathscr{K}}$ are the "classical" Toeplitz operators.

EXAMPLE 2. Here we set forth a class of Riesz systems all related to the Riesz system $(\widetilde{\mathscr{K}}, \widetilde{\boldsymbol{R}}, \widetilde{\mathscr{K}})$ of Example 1 . Let $F$ be any finite subset of the non-negative integers, and consider the subspace $\mathscr{M}_{F} \subset \widetilde{\mathscr{K}}_{\text {span- }}$ ned by the orthonormal vectors $\left\{z^{n}: n \geqq 0, n \notin F\right\}$. It is an easy exercise to verify that $\left(\widetilde{\mathbb{E}}, \widetilde{R}, \mathscr{M}_{F}\right)$ is a Riesz system, and for future reference we denote such a Riesz system by $R_{F}$.

Further examples of Riesz systems related to $(\widetilde{\mathbb{E}}, \widetilde{R}, \widetilde{\mathscr{K}})$ can be obtained by considering any subspace $\mathscr{N}$ of $\bigvee\left\{z^{n}: n=1,2, \cdots N\right\}$ and replacing the subspace $\widetilde{\mathscr{K}}_{\text {by }}$ the subspace $\widetilde{\mathscr{K}} \ominus \mathscr{N}$.

EXAmpLE 3. Let $G$ be any (nontrival) subgroup of the additive group of real numbers, and regard $G$ as a discrete topological group. Let $G$ be the compact character group of $G$, let $\nu$ denote normalized Haar measure on $G$, and let $R$ be the maximal abelian v. N. algebra of multiplications by bounded measurable functions on the Hilbert space $L^{2}(\nu, \mathcal{G})$. The elements $r \in G$, regarded as functions $r(\mathrm{\chi})$ on $\hat{G}$, form an orthonormal basis for $L^{2}(\nu, \widehat{G})$. Thus an analogue of the subspace $\widetilde{\mathscr{K}}$ in Example 1 can be obtained by defining $\mathscr{K}=\Lambda\{r(\mathrm{x}): r \in G, r \leqq 0\}$. It has been shown by Helson and Lowdenslager [5, pp. 177-178, 195] that $\left(L^{2}(\nu, G), R, \mathscr{K}\right)$ is a Riesz system. It should be noted that if $G$ is taken to be the additive group of real numbers itself, then the Hilbert space $L^{2}\left(\nu, G^{2}\right)$ is nonseparable; thus there exist Riesz systems associated with nonseparable Hilbert spaces.

EXAmple 4. Let $(S, \mu, \mathscr{S})$ be any finite measure space, and let $R$ be the v. N. algebra of all multiplications by bounded measurable functions on the Hilbert space $L^{2}(\mu, S)$. Let $\mathscr{K}$ be any subspace of $L^{2}(\mu, S)$ with the property that if $f \neq 0 \in \mathscr{K} \cup \mathscr{K}^{+}$, then $f$ vanishes on at most a set 
of measure zero. It is known [9] that such a v. N. algebra $R$ is maximal abelian, and it follows immediately that $\left(L^{2}(\mu, S), R, \mathscr{K}\right)$ is a Riesz system.

We remark that the existence of such a subspace $\mathscr{K}$ imposes some restrictions on the measure space $(S, \mu, \mathscr{S})$. In particular, it follows from Proposition 3.3 below that $(S, \mu, \mathscr{S})$ must be nonatomic and homogeneous.

We also remark that it can be shown that every Riesz system is equivalent (in a sense to be defined in $\$ 4$ ) to a Riesz system of the above type. That this is true follows from the fact that $R$ always has a cyclic vector and the standard representation theory for maximal abelian $v$. N. algebras [8]. Thus if one is content to consider Riesz systems on separable spaces, there is no loss of generality in taking $(S, \mu, \mathscr{S})$ to be Lebesgue measure on the interval $[0,1]$ and $R$ to be $L^{\infty}(\mu, S)$.

3. The spectral inclusion theorem. Throughout this section we suppose as given some fixed but arbitrary Riesz system $(\mathscr{H}, \boldsymbol{R}, \mathscr{K})$. Recall that $P$ denotes the projection of $\mathscr{H}$ onto $\mathscr{K}$. We first obtain some preliminary results that lead to the spectral inclusion theorem, and one piece of additional notation is needed. If $\mathscr{M}$ is any linear manifold in $\mathscr{E}$, then the closure of $\mathscr{M}$ will be denoted by $[\mathscr{M}]$.

Lemma 3.1. If $L \in R$ and $[L \mathscr{H}] \neq \mathscr{H}$, then $[L \mathscr{H}]=[L \mathscr{K}]=\left[L \mathscr{K}^{+}\right]$.

Proof. Clearly $[L \mathscr{K}] \subset[L \mathscr{H}]$. Suppose now that $f \in[L \mathscr{L}]$ is such that $f$ is orthogonal to $[L \mathscr{K}]$. Let $E$ be the projection on the subspace $[L \mathscr{Z}]$. Then one knows that $E \in R$, and that $(I-E) f=0$. Now for each $k \in \mathscr{K},\left(L^{*} f, k\right)=(f, L k)=0$. Thus $L^{*} f \in \mathscr{K}^{\perp}$, and since $(I-E) f$ $=0,(I-E) L^{*} f=L^{*}(I-E) f=0$. Since $I-E$ is a nonzero element of $\boldsymbol{R}$ and $\mathscr{K}^{+}$is a weak Riesz subspace for $R, L^{*} f=0$. Thus $f$ is a null vector for $L^{*}$, which implies that $f$ is orthogonal to $[L \mathscr{Z}]$, so that $f=0$. This proves that $[L \mathscr{K}]=[L \mathscr{H}]$, and the result for $\left[L \mathscr{K}^{{ }^{\perp}}\right]$ follows from the symmetry of the assumptions on the subspaces $\mathscr{K}$ and $\mathscr{K}^{+}$.

We remark that Lemma 3.1 actually characterizes Riesz systems in the sense that if $\mathscr{H}$ is an infinite-dimensional Hilbert space, $R$ is a maximal abelian v. N. algebra on $\mathscr{H}$, and $\mathscr{K}$ is a subspace of $\mathscr{H}$, then $(\mathscr{H}, R, \mathscr{K})$ is a Riesz system if and only if $E \mathscr{H}=[E \mathscr{K}]=\left[E \mathscr{K}^{\perp}\right]$ for every nonzero projection $E<1$ in $R$.

Proposition 3.2. If $0 \neq L \in R$ and $[L \mathscr{H}] \neq \mathscr{H}$, then $[P L \mathscr{K}]=\mathscr{K}$.

Proof. Clearly $[P L \mathscr{K}] \subset \mathscr{K}$, and it follows easily from Lemma 3.1 that $[P L \mathscr{K}]=[P L \mathscr{A}]$. Thus suppose that $k \in \mathscr{K}$ is such that $k$ is orthogonal to $[P L \mathscr{H}]$. Then for all $h \in \mathscr{H},\left(L^{*} k, h\right)=(k, L h)=0$, so that $L^{*} k=0$. Since $0 \neq L^{*} \in R, k=0$. 
Proposition 3.3. The maximal abelian $v$. $N$. algebra $R$ contains no (nonzero) minimal projections, and the subspaces $\mathscr{K}$ and $\mathscr{K}^{\perp}$ are both infinitedimensional.

Proof. Suppose that $E$ is a minimal projection in $R$. Then $E$ must project onto a one-dimensional subspace of $\mathscr{H}$, since any minimal projection in a maximal abelian v. N. algebra has this property. From Lemma 3.1 we know that $E \mathscr{H}=[E \mathscr{K}]=\left[E \mathscr{K}^{\perp}\right]$, and since $E$ must map $\mathscr{K}$ and $\mathscr{K}^{\perp}$ in a one-to-one fashion, it follows that $\operatorname{dim} \mathscr{K}=\operatorname{dim}[E \mathscr{K}]$ $=\operatorname{dim}\left[E \mathscr{K}^{1}\right]=\operatorname{dim} \mathscr{K}^{\perp}=\operatorname{dim} E \mathscr{H}=1$. Thus $\mathscr{H}=\mathscr{K} \oplus \mathscr{K}^{\perp}$ is twodimensional, a contradiction. Thus $R$ contains no minimal projections, and therefore no projections of finite rank. The fact that $E \mathscr{H}=[E \mathscr{K}]$ $=\left[E \mathscr{K}^{\perp}\right]$ then forces $\mathscr{K}$ and $\mathscr{K}^{\perp}$ to be infinite-dimensional subspaces of $\mathscr{H}$. Furthermore, it is not hard to show that $\operatorname{dim}(E \mathscr{K})=\operatorname{dim}[E \mathscr{K}]$ $=\operatorname{dim} \mathscr{K}$ for all nonzero projections $E<I$ in $R$. Thus if $R$ is represented as the multiplication algebra of $L^{2}$ of a finite measure space, then the measure space must be nonatomic and homogeneous.

Before continuing to the proof of the spectral inclusion theorem, we introduce the following terminology. If $A$ is any operator, we denote by $\sigma(A)$ the spectrum of $A$ and by $\pi(A)$ the approximate point spectrum of $A$. Furthermore, if $L \in R$ (so that $L$ is a (GL) operator), then the (GT) operator $P L$ on $\mathscr{K}$ will be denoted by $T_{L}$.

Theorem 1. If $L$ is a $(G L)$ operator, then $\sigma(L) \subset \sigma\left(T_{L}\right)$, and in fact, $\sigma(L) \subset \pi\left(T_{L}\right)$.

Proof. Since $T_{L-\lambda I_{\mathscr{H}}}=T_{L}-\lambda I_{\mathscr{K}}$, it suffices to show that if $0 \in \sigma(L)$, then $0 \in \pi\left(T_{L}\right)$. Also if $L=0$, then clearly $T_{L}=0$, so that we can assume that $L \neq 0$. Let $\epsilon>0$ be given, and choose a spectral projection $E \in R$ of $L$ such that $0<E<I_{\mathscr{H}}$ and such that $\|E L\|<\epsilon$. Since $R$ contains no minimal projections, there exists a projection $F \in R$ such that $I>F$ $>I-E$. Let $f$ be any unit vector in $\mathscr{H}$ such that $F E f=f$. Since we know from Lemma 3.1 that $[F \mathscr{K}]=F \mathscr{H}$, it results that there exists a sequence of vectors $k_{n} \in \mathscr{K}$ such that $\left\|F k_{n}-F f\right\| \rightarrow 0$. We consider first the case that the sequence $\left\{\left\|k_{n}\right\|\right\}$ is bounded. Then

$$
\begin{aligned}
\left\|T_{L} k_{n}\right\|=\left\|P L k_{n}\right\| & \leqq\left\|L k_{n}\right\|=\left\|L F k_{n}-L F f+L F f+L(I-F) k_{n}\right\| \\
& \leqq\|L\|\left\|F k_{n}-F f\right\|+\|L E F f\|+\left\|L E(I-F) k_{n}\right\| \\
& \leqq\|L\|\left\|F k_{n}-F f\right\|+\epsilon+\epsilon\left\|k_{n}\right\| .
\end{aligned}
$$

Also

$$
\begin{aligned}
\left\|k_{n}\right\| & \geqq\left\|F k_{n}\right\|=\left\|F f-\left(F f-F k_{n}\right)\right\| \\
& \geqq\|F f\|-\left\|F f-F k_{n}\right\|=I-\left\|F f-F k_{n}\right\| .
\end{aligned}
$$


Since $\left\|F k_{n}-F f\right\| \rightarrow 0$, for sufficiently large $n$ we have $\left\|T_{L} k_{n}\right\| \leqq 3 \epsilon\left\|k_{n}\right\|$, which proves that $0 \in \pi\left(T_{L}\right)$.

On the other hand, if the sequence $\left\{\left\|k_{n}\right\|\right\}$ is unbounded, choose a subsequence $\left\{k_{n(t)}\right\}$ such that $\left\|k_{n(t+1)}-k_{n(t)}\right\| \geqq 1$ for all $t$. Upon setting $h_{t}$ $=\left\|k_{n(t+1)}-k_{n(t)}\right\|^{-1}\left(k_{n(t+1)}-k_{n(t)}\right)$, we have

$$
\begin{aligned}
\left\|T_{L} h_{t}\right\| & \leqq\left\|L h_{t}\right\|=\left\|L E h_{t}+L(I-E) h_{t}\right\| \\
& \leqq \epsilon+\left\|L(I-E) F h_{t}\right\| \leqq \epsilon+\|L\|\left\|F h_{t}\right\| \\
& \leqq \epsilon+\|L\|\left\|F k_{n(t+1)}-F k_{n(t)}\right\| .
\end{aligned}
$$

Since $\left\|F k_{n(t+1)}-F k_{n(t)}\right\| \rightarrow 0$, for large enough $t$ we have $\left\|T_{L} h_{t}\right\|<2 \epsilon$, so that again $0 \in \pi\left(T_{L}\right)$.

It is interesting to note that in the argument just completed the unit vectors which $L$ made small were taken from the subspace $\mathscr{K}$.

We now settle down to exploiting the above result. The spectral radius of an operator $A$ will be denoted by $\rho(A)$.

Proposition 3.4. The mapping $L \rightarrow T_{L}$ is linear and one-to-one and preserves adjoints, spectral radii, and norms.

Proof. That the mapping is linear and adjoint-preserving is completely obvious, and its one-to-oneness will follow from the fact that it is normpreserving. Now from above we know that $\rho(L) \leqq \rho\left(T_{L}\right)$. Therefore $\|L\|$ $=\rho(L) \leqq \rho\left(T_{L}\right) \leqq\left\|T_{L}\right\|$. Also since for $f \in \mathscr{K},\left\|T_{L} f\right\|=\|P L f\| \leqq\|L f\|$, we know that $\left\|T_{L}\right\| \leqq\|L\|$, and the argument is complete.

Proposition 3.5. If $L$ is a $(G L)$ operator, then the closure of the numerical range of $L$ is identical with the closure of the numerical range of $T_{L}$.

Proof. Let $W(L)$ denote the numerical range of $L$. Since $\left(T_{L} f, f\right)=(L f, f)$ for $f \in \mathscr{K}, W\left(T_{L}\right) \subset W(L)$ and $\left[W\left(T_{L}\right)\right]^{\mathrm{cl}} \subset[W(L)]^{\mathrm{cl}}$. Also $\left[W\left(T_{L}\right)\right]^{\mathrm{cl}}$ contains the spectrum of $T_{L}$ and thus the spectrum of $L$. Since $\left[W\left(T_{L}\right)\right]^{\text {cl }}$ is closed and convex, it must contain the closed convex hull of $\sigma(L)$, i.e., it contains $[W(L)]^{\mathrm{cl}}$.

Note that it follows easily that $\left[W\left(T_{L}\right)\right]^{\text {cl }}$ is the closed convex hull of $\sigma\left(T_{L}\right)$, and that $L$ is positive semi-definite if and only if $T_{L}$ is also.

Proposition 3.6. If $L$ is a $(G L)$ operator that is not a scalar, then $L$ and $T_{L}$ have no proper value in common.

Proof. Since $T_{L+\lambda I_{\mathscr{H}}}=T_{L}+\lambda I_{\mathscr{K}}$, it suffices to show that if 0 is a proper value for an $L \neq \lambda I$ in $R$, then 0 is not a proper value for $T_{L}$. Thus let $E \in R$ be a spectral projection for $L$ such that $0<E<I_{\mathscr{H}}$ and such that $E L=0$. Now suppose that $k \in \mathscr{K}$ satisfies $T_{L} k=0$. Then $P(L k)=0$ so that $L k \in \mathscr{K}^{\perp}$. Since $E(L k)=0, L k=0$, and since $k \in \mathscr{K}$ and $L \neq 0$, $k=0$. 
Corollary 3.7. The only compact $(G T)$ operator is 0 .

Proof. Suppose that $T_{L}$ is compact and nonzero. Then $L \neq 0$ and $\sigma(L)$ contains at least one nonzero number $\lambda$. It follows from Theorem 1 that $\lambda \in \sigma\left(T_{L}\right)$, and since $T_{L}$ is compact and $\sigma(L) \subset \sigma\left(T_{L}\right), \lambda$ must be an isolated proper value for each of the operators $T_{L}$ and $L$. But this contradicts Proposition 3.6 , so that $T_{L}=0$.

The spectral theory of (GT) operators is now sufficiently far advanced to enable us to reprove several additional results from [2]. For example, Theorem 10 of [2] along with its Corollaries 1, 2, and 3 are valid of (GT) operators. Somewhat more interesting is the valid generalization of Theorem 12: Every nonscalar $(G L)$ operator $L$ is a minimal normal dilation of $T_{L}$.

Since the proofs of these results are essentially the same as those in [2], we omit them and continue to a discussion of some results of [2] for which different arguments had to be improvised. A (GL) operator $L$ and its associated (GT) operator $T_{L}$ are said to be analytic if $L \mathscr{K} \subset \mathscr{K}$ and to be co-analytic if $L \mathscr{K}^{\perp} \subset \mathscr{K}^{\perp}$ It follows that $L$ (resp. $T_{L}$ ) is analytic if and only if $L^{*}$ (resp. $\left.T_{L}^{*}\right)$ is co-analytic.

Lemma 3.8. If $L \neq \lambda I$ is any $(G L)$ operator and $Q$ is any projection such that $0<Q<I_{\mathscr{K}}$ and such that $Q \leqq P$ or $P \leqq Q$, then $L Q \neq Q L$.

Proof. We consider the case $P \leqq Q$; the case $Q \leqq P$ is proved similarly. Suppose $L Q=Q L$, and let $E \in R$ be a spectral projection for $L$ such that $0<E<I$. Let $\mathscr{M}=Q \mathscr{H}$ and observe that $\mathscr{K} \subset \mathscr{M}$. Since $E Q=Q E$, $E \mathscr{M} \subset \mathscr{M}$, and from Lemma 3.1 one knows that $E \mathscr{H}=[E \mathscr{K}]$. Thus $E \mathscr{H}=[E \mathscr{K}] \subset[E \mathscr{M}] \subset \mathscr{M}$. Since also $I-E \in R$ and $(I-E) \mathscr{M}$ $\subset \mathscr{M}$, it follows similarly that $(I-E) \mathscr{H} \subset \mathscr{M}$, which implies that $\mathscr{M}$ $=\mathscr{H}$, a contradiction.

Corollary 3.9. The only $(G L)$ operators that are both analytic and coanalytic are the scalars.

Proof. If $L$ is analytic and co-analytic, the $L P=P L$ and the result follows from the preceding lemma.

THeorem 2. $A(G T)$ operator $T_{L}$ is an isometry if and only if $L$ is an analytic unitary operator. Furthermore such a $T_{L}$ is either a scalar of modulus one or a pure isometry $\left({ }^{1}\right)$.

Proof. If $L$ is an analytic, unitary operator it is clear that $T_{L}$ is an isometry. Thus, going the other way, suppose $T_{L}$ is an isometry. We know from Proposition 3.4 that $\|L\|=\left\|T_{L}\right\|=1$, so that for each $k \in \mathscr{K}$,

( ${ }^{1}$ ) An isometry $S$ is pure if there is no reducing subspace for $S$ on which $S$ acts as a unitary operator [1]. 
$\|L k\| \leqq\|k\|=\left\|T_{L} k\right\|=\|P L k\| \leqq\|L k\|$. Thus for each $k \in \mathscr{K},\|P(L k)\|$ $=\|L k\|=\|k\|$ which implies that $P L k=L k$ since $P$ is a projection. Thus $L \mathscr{K} \subset \mathscr{K}$, and $L$ is analytic. If $L$ is not a unitary operator, then a spectral projection $E \in R$ of $L$ can be chosen so that $E \neq 0$ and $\|E L\|<1$. If $0 \neq k \in \mathscr{K}$, then

$$
\begin{aligned}
\|k\|^{2} & =\|L k\|^{2}=\|L E k\|^{2}+\|(I-E) L k\|^{2} \\
& \leqq\|L E\|^{2}\|E k\|^{2}+\|L\|^{2}\|(I-E) k\|^{2} \\
& <\|E k\|^{2}+\|(I-E) k\|^{2}=\|k\|^{2},
\end{aligned}
$$

which is a contradiction. Thus $L$ must be an analytic unitary operator. Now suppose that $T_{L}$ is not a pure isometry. Then there is a nonzero subspace $\mathscr{M} \subset \mathscr{K}$ such that $\mathscr{M}$ reduces $T_{L}$ and such that $T_{L} \mid \mathscr{M}$ is a unitary operator. Since $L$ is analytic, it follows that $L \mathscr{M} \subset \mathscr{M}$ and that $L\left|\mathscr{M}=T_{L}\right| \mathscr{M}$ is unitary. Thus the restriction of $L$ to the invariant subspace $\mathscr{M}$ is a normal operator, and it follows that $\mathscr{M} \subset \mathscr{K}$ reduces $L$. By virtue of Lemma 3.8, $L$ is forced to be a scalar of modulus one, so that $T_{L}$ is also a scalar of modulus one, and the argument is complete.

The final result to be proved in this section is that the spectrum of every analytic (GT) operator is connected. For this purpose we need the following lemma.

Lemma 3.10. The collection of analytic Toeplitz operators associated with any Riesz system forms a semi-simple commutative Banach algebra under the operator norm. Furthermore, the operator spectrum of any analytic (GT) operator is the same as its spectrum regarded as an element in this Banach algebra.

Proof. Let $A$ be the collection of analytic (GT) operators associated with a Riesz system $(\mathscr{H}, R, \mathscr{K})$. It is clear that if $T_{L_{1}}$ and $T_{L_{2}}$ are in $A$, then $T_{L_{1}} T_{L_{2}}=T_{L_{1} L_{2}} \in A$, so that $\boldsymbol{A}$ is at least a commutative operator algebra. Now suppose that $\left\{T_{L_{n}}\right\}$ is a sequence of analytic Toeplitz operators converging to an operator on $\mathscr{K}$. Then the sequence $\left\{T_{L_{n}}\right\}$ is Cauchy in the uniform operator topology, and since the map $L \rightarrow T_{L}$ is norm preserving by Proposition 3.4, the sequence $\left\{L_{n}\right\}$ is also Cauchy. Thus the sequence $\left\{L_{n}\right\}$ converges in the uniform operator topology to an analytic (GL) operator $L \in R$, and it follows that $T_{L_{n}} \rightarrow T_{L}$. Thus $A$ is a commutative Banach algebra, and is semi-simple because no $T_{L}$ is quasi-nilpotent.

Now let $\Sigma\left(T_{L}\right)$ denote the spectrum of an analytic (GT) operator $T_{L}$ regarded as an element of the Banach algebra $A$. Since $I_{\mathscr{K}} \in A$, it is obvious that $\sigma\left(T_{L}\right) \subset \Sigma\left(T_{L}\right)$. Since $T_{L}-\lambda I_{\mathscr{K}}=T_{L-\lambda I_{\mathscr{E}}}$, to prove $\Sigma\left(T_{L}\right)$ $C \sigma\left(T_{L}\right)$ it suffices to show that if $0 \notin \sigma\left(T_{L}\right)$, then $0 \notin \Sigma\left(T_{L}\right)$. Thus suppose that $T_{L}$ is an invertible operator on $\mathscr{K}$. It follows from Theorem 1 that 
$L$ is invertible on $\mathscr{H}$, and one knows that $L^{-1} \in R$. For $k \in \mathscr{K}, T_{L^{-1}} T_{L} k$ $=T_{L^{-1}} P L k=T_{L^{-1}} L k=P L^{-1} L k=P k=k$ since $L$ is analytic. Thus $T_{L^{-1}}$ is a left inverse for the invertible operator $T_{L}$ and it follows that $\left(T_{L}\right)^{-1}=T_{L^{-1}}$. Thus for each $k \in \mathscr{K}, L P L^{-1} k=T_{L} T_{L^{-1}} k=k$, so that $P L^{-1} k=L^{-1} k$. Thus $L^{-1} k \in \mathscr{K}$, which proves that $L^{-1}$ is analytic and that $0 \notin \Sigma\left(T_{L}\right)$.

Theorem 3. The spectrum of every analytic $(G T)$ operator $T_{L}$ is connected.

Proof. By the above lemma, it suffices to show that the spectrum of $T_{L}$ regarded as an element of $\boldsymbol{A}$ is connected. The Gel' fand theory tells us that $T_{L}$ can be regarded as a continuous complex-valued function $t(\cdot)$ on the compact maximal ideal space $X$ of $A$, and the spectrum of $T_{L}$ is then the range of the function $t(\cdot)$. Since the continuous image of a connected set is connected, it suffices to prove that the maximal ideal space $X$ is connected. By the well-known theorem of Šilov [10], $X$ will be connected provided $A$ contains no idempotents other than 0 and $I$. Thus suppose that $E=T_{L_{1}} \in A$ is an idempotent analytic (GT) operator. Then by the spectral inclusion theorem, the analytic (GL) operator $L_{1}$ must be a projection. But it follows easily from Corollary 3.9 that the only Hermitian analytic (GL) operators are the real scalars, so that $L_{1}=0$ or $L_{1}=I_{\mathscr{\sharp}}$, and the proof is complete.

4. Nonequivalent Riesz systems. In this section we study ways in which Riesz systems can differ. Two Riesz systems $(\mathscr{H}, R, \mathscr{K})$ and $\left(\mathscr{H}_{1}, \boldsymbol{R}_{1}, \mathscr{K}_{1}\right)$ are said to be equivalent if and only if there is a Hilbert space isomorphism $\phi$ of $\mathscr{H}$ onto $\mathscr{H}_{1}$ such that $\phi(\mathscr{K})=\mathscr{K}_{1}$ and such that $\phi \boldsymbol{R} \phi^{-1}=\boldsymbol{R}_{1}$. We first show that there are nonequivalent Riesz systems on a separable Hilbert space. For this purpose, note that if $(\mathscr{H}, R, \mathscr{K})$ and $\left(\mathscr{H}_{1}, \boldsymbol{R}_{1}, \mathscr{K}_{1}\right)$ are any Riesz systems equivalent under an isomorphism $\phi: \mathscr{H} \rightarrow \mathscr{H}_{1}$ and $T$ is a (GT) operator associated with $(\mathscr{H}, R, \mathscr{K})$, then $\phi \mid \mathscr{K}$ carries $T$ onto a (GT) operator $T_{1}$ associated with $\left(\mathscr{H}_{1}, \mathbf{R}_{1}, \mathscr{K}_{1}\right)$ that is unitarily equivalent to $T$. This elementary observation can be used to prove that some of the Riesz systems $R_{F}$ set forth in Example 2 are not equivalent.

We continue to denote the original Riesz system of Example 1 by $(\widetilde{\mathscr{K}}, \widetilde{R}, \widetilde{\mathscr{K}})$ and the projection of $\widetilde{\mathscr{K}}$ onto $\widetilde{\mathscr{K}}$ by $\widetilde{P}$. For each positive integer $n$, let $F(n)$ be the set of integers $F(n)=\{1,3, \cdots,(2 n-3),(2 n-1)\}$, and let $Q_{n}$ be the projection of $\widetilde{\mathscr{E}}$ onto the subspace $\mathscr{M}_{F(n)}$ (in the terminology of Example 2). Recall that the maximal abelian v. N. algebra $R$ associated with each Riesz system $R_{F(n)}$ is just $\widetilde{R}$, the algebra of multiplications by bounded measurable functions on $\widetilde{\mathscr{E}}=L_{2}(\mu, C)$. Also note that if $L$ is any operator in $\widetilde{R}$, then the (GT) operator associated with $L$ and $R_{F(n)}$ can be written as $Q_{n} L\left|\mathscr{M}_{F(n)}=Q_{n} \widetilde{P} L\right| \mathscr{M}_{F(n)}$. Now it is known 
[4] that every nonzero Hermitian Toeplitz operator must have a trivial null space. Thus if $L=L^{*} \in \widetilde{\boldsymbol{R}}, \widetilde{P L}$ must map $\mathscr{M}_{F(n)}$ in a one-to-one fashion since $\mathscr{M}_{F(n)} \subset \mathscr{\mathscr { K }}$. It follows that if $f \in \mathscr{M}_{F(n)}$ and $Q_{n} L f=Q_{n} \widetilde{P} L f=0$, then $\widetilde{P L f} \in\left(P-Q_{n}\right) \widetilde{\mathscr{H}}$. Since $\left(P-Q_{n}\right) \widetilde{\mathscr{H}}$ is an $n$-dimensional subspace of $\widetilde{E}$ we have proved the following lemma.

LEMma 4.1. No Hermitian (GT) operator associated with the Riesz system $\left(\widetilde{\mathbb{E}}, \widetilde{\boldsymbol{R}}, \mathscr{M}_{F(n)}\right)$ has a null space whose dimension exceeds $n$.

Now let $n_{0}>0$ be arbitrary but fixed, and consider the operator $L \in \widetilde{\boldsymbol{R}}$ that multiplies every vector $f \in \mathscr{\&}$ by the function $\phi(z)=z+z^{-1}$. Since $\phi$ is real on the unit circle $C, L$ is Hermitian, so that the (GT) operator $Q_{n_{0}} L \mid \mathscr{M}_{F\left(n_{0}\right)}$ associated with the Riesz system $\left(\widetilde{\mathscr{H}}, \widetilde{\boldsymbol{R}}, \mathscr{M}_{F\left(n_{0}\right)}\right)$ is also Hermitian. $A$ simple calculation shows that the orthonormal vectors $f_{k}(z)$ $=z^{k}, k=0,2, \cdots, 2 n_{0}-2$, in $\mathscr{M}_{F\left(n_{0}\right)}$ are all in the null space of ${\underline{n_{0}}}_{\tilde{R}} L \mid \mathscr{M}_{F\left(n_{0}\right)}$, so that there is a Hermitian (GT) operator associated with $\left(\widetilde{\mathscr{E}}, \widetilde{\boldsymbol{R}}, \mathscr{M}_{F\left(n_{0}\right)}\right)$ that has an $n_{0}$-dimensional null space. This fact together with Lemma 4.1 yields:

THEOREM 4. If $m$ and $n$ are distinct positive integers, then the Riesz systems $R_{F(m)}$ and $R_{F(n)}$ are not equivalent. Furthermore no Riesz system $R_{F(n)}$ is equivalent to $(\widetilde{\mathscr{H}}, \widetilde{\boldsymbol{R}}, \widetilde{\mathscr{K}})$.

Although in general the question of whether two Riesz systems are equivalent seems to be a difficult one, we are able to show that at least one of the Riesz systems of Example 3 is not equivalent to $(\widetilde{\mathscr{H}}, \widetilde{\boldsymbol{R}}, \widetilde{\mathscr{K}})$. To do this, the following lemma will be needed.

Lemma 4.2. Let $\mathscr{\mathscr { E }}=L_{2}(\mu, C)$ be the Hilbert space of Example 1 , and let $\left\{f_{n}\right\}_{n=1}^{\infty}$ be a bounded sequence of vectors in the subspace $\widetilde{\mathscr{K}} \subset \widetilde{\mathscr{H}}$. Let $f_{n}(z)$ be the analytic extension of $f_{n}$ to the open unit disc $\mathscr{D}$, and suppose that the sequence $\left\{f_{n}(z)\right\}$ converges subuniformly on $\mathscr{D}$ to an analytic function $f(z)$ that is the analytic extension of a vector $f \in \widetilde{\mathscr{K}}$ to $\mathscr{D}$. Then the sequence of vectors $\left\{f_{n}\right\}$ converges in the weak topology of $\widetilde{\mathscr{K}}$ to $f$.

Sketch of the proof. Let $\left\{e_{k}(z)=z^{k}:|z|=1, k \geqq 0\right\}$ denote the orthonormal basis for $\widetilde{\mathscr{K}}$ considered in Example 1. The Fourier coefficients of each vector $f_{n}$ (resp. the vector $f$ ) are the Taylor coefficients of the analytic function $f_{n}(z)$ (resp. $f(z)$ ) and the subuniform convergence of the sequence $f_{n}(z)$ to $f(z)$ guarantees that for each $m \geqq 0$, the sequence of $m$ th Taylor coefficients of the $f_{n}(z)$ converges to the $m$ th Taylor coefficient of $f(z)$. It follows that $\left(f_{n}, e_{k}\right) \rightarrow\left(f, e_{k}\right)$ for all $k \geqq 0$, and thus that $\left(f_{n}, g\right) \rightarrow(f, g)$, where $g$ runs over a dense set of vectors in $\widetilde{\mathscr{K}}$. In the presence of the boundedness of the sequence $\left\{f_{n}\right\}$, this is enough to enable one to conclude that the sequence $\left\{f_{n}\right\}$ converges to $f$ in the weak topology of $\widetilde{\mathscr{K}}$. 
Now let $G$ denote the additive group of rational numbers, and let $\widehat{G}, \boldsymbol{R}$ and $\mathscr{K}$ be as defined in Example 3.

TheORem $5\left(^{2}\right)$. The Riesz systems $\left(L^{2}(\nu, \widehat{G}), \boldsymbol{R}, \mathscr{K}\right)$ and $(\tilde{\mathscr{E}}, \widetilde{\boldsymbol{R}}, \tilde{\mathscr{K}})$ are not equivalent.

Proof. We assume that these Riesz systems are equivalent, and denote by $\phi$ a unitary isomorphism from $L^{2}(\nu, \hat{G})$ onto $\widetilde{\mathscr{E}}$ that implements this equivalence. Let $G^{+}$denote the subset of $G$ consisting of all nonnegative rational numbers, and let $r \in G^{+}$. Multiplication by the bounded measurable function $r(\mathrm{x})$ on $\hat{G}$ determines an analytic unitary (GL) operator $L_{r}$ on $L^{2}(\nu, \hat{G})$, and if $e$ denotes the function identically one on $\hat{G}$, the vectors $\left\{L_{r} e\right\}_{r \in G^{+}}$form an orthonormal basis for the subspace $\mathscr{K}$. For each $r \in G^{+}$, denote by $L_{h_{r}}$ the Laurent operator $\phi L_{r} \phi^{-1} \in \widetilde{R}$, and observe that if $f=\phi e$, then $\left\{L_{h_{r}} f\right\}_{r \in G^{+}}$forms an orthonormal basis for $\widetilde{\mathscr{K}}$. Since $L_{h_{r}}$ is an analytic unitary Laurent opertor, $L_{h_{r}}$ must be multiplication on $L^{2}(\mu, C)$ by an inner function - say $h_{r}$. (See [6, p. 62] for the definition of inner function.) Now for each $r \in G^{+}$, let $h_{r}(z)$ denote the extension of $h_{r}$ to a bounded analytic function defined on the open unit disc $\mathscr{D}$ in the complex plane. Since $h_{r_{1}} \cdot h_{r_{2}}=h_{r_{1}+r_{2}}$ it follows that for each $r \in G^{+}$and each $z \in \mathscr{D}, h_{r}(z)=\left[h_{r / n}(z)\right]^{n}$ for each positive integer $n$. Thus for $z \in \mathscr{D}$, $h_{r}(z) \neq 0$ (otherwise, $h_{r}(z)$ would have a zero of arbitrarily large multiplicity), and each $h_{r}(z)$ can be written $h_{r}(z)=e^{i \theta_{r}} k_{r}(z)$ on $\mathscr{D}$ where $k_{r}(z)$ is a singular inner function [6, p. 67] satisfying $k_{r}(0)>0$. If $k_{r}$ denotes the (essentially unique) bounded measurable function defined on the unit circle $C$ whose analytic extension to $\mathscr{D}$ is $k_{r}(z)$, then $\left(k_{r} f, f\right)=e^{-i \theta_{r}}\left(L_{h_{2}} f, L_{h_{r}} f\right)$ $=0$. On the other hand, if we denote the unique (on $\mathscr{D}) n$th root of the function $k_{1}(z)$ that is positive at the origin by $\left[k_{1}(z)\right]^{1 / n}$, it must be that $k_{1 / n}(z)=\left[k_{1}(z)\right]^{1 / n}$. It follows easily from the representation theorem for singular inner functions $[6$, p. 66], that the sequence of analytic function $\left\{k_{1 / n}(z)\right\}$ converges uniformly on compact subsets of $\mathscr{D}$ to the analytic function $I(z) \equiv 1$. If $f(z)$ denotes the analytic extension to $\mathscr{D}$ of the function $f \in \mathscr{\mathscr { K }}$, then the sequence $\left\{k_{1 / n}(z) f(z)\right\}$ converges subuniformly on $\mathscr{D}$ to $f(z)$ and the corresponding sequence $\left\{k_{1 / n} f\right\} \in \mathscr{\mathscr { K }}$ is bounded. It thus follows from Lemma 4.2 that the sequence of vectors $k_{1 / n} f \in \mathscr{\mathscr { K }}$ converges weakly to $f$, and thus for $n$ sufficiently large, $\left(k_{1 / n} f, f\right) \neq 0$. This contradicts an earlier equation and completes the proof.

Actually the above proof can be used to show that a large class of subgroups of the reals yield Riesz systems not equivalent to $(\widetilde{\mathscr{E}}, \widetilde{\boldsymbol{R}}, \widetilde{\mathscr{K}})$.

The above theorem completes our results on the nonequivalence of Riesz systems, and we now want to look at the question the other way

${ }^{2}$ ) One of the central ideas in the proof of this theorem was kindly pointed out to us by Eric Nordgren. 
around and ask what additonal structure need be added to a Riesz system to force it to be equivalent to $(\widetilde{\mathscr{E}}, \widetilde{\boldsymbol{R}}, \widetilde{\mathscr{K}})$ ? In this connection we can prove the following theorem.

Theorem 6. Suppose that associated with the Riesz system ( $\mathscr{H}, \boldsymbol{R}, \mathscr{K})$ there is a (GT) operator $U$ that is a unilateral shift of multiplicity one. Then $(\mathscr{H}, \boldsymbol{R}, \mathscr{K})$ is equivalent to $(\widetilde{\mathscr{H}}, \widetilde{\boldsymbol{R}}, \widetilde{\mathscr{K}})$.

Proof. To say that $U$ is a unilateral shift of multiplicity one means that there is an orthonormal basis $\left\{f_{n}\right\}_{n=0}^{\infty}$ for $\mathscr{K}$ such that $U f_{k}=f_{k+1}$ for $k=0,1,2, \cdots$. Since $U$ is by assumption a (GT) operator, there exists an $L \in \boldsymbol{R}$ such that $U=P L \mid \mathscr{K}$. Since $U$ is an isometry, it follows from Theorem 2 that $L$ must be an analytic unitary operator. Now for all positive integers $k$, let $f_{-k}=L^{-k}\left(f_{0}\right)$. It follows that the family $\left\{f_{k}\right\}_{k=-\infty}^{\infty}$ is an orthonormal family of vectors in $\mathscr{H}$ and that the subspace $\mathscr{M} \subset \mathscr{H}$ spanned by the $f_{k},-\infty<k<\infty$, is a reducing subspace for $L$. Since $\mathscr{M} \supset \mathscr{K}$, it follows from Lemma 3.8 that $\mathscr{M}=\mathscr{K}$; in other words, the family $\left\{f_{k}\right\}_{k=-\infty}^{\infty}$ forms an orthonormal basis for $\mathscr{H}$. We define an isomorphism $\phi$ from the Hilbert space $\mathbb{Z}$ onto $\widetilde{\mathscr{E}}$ by defining $\phi\left(f_{n}\right)=z^{n} \in \widetilde{\mathscr{K}}$ for each integer $n$. Then clearly $\phi(\mathscr{K})=\widetilde{\mathscr{K}}$, and it suffices to show that $\phi \boldsymbol{R} \phi^{-1}=\widetilde{\boldsymbol{R}}$. We have established that $L$ is a bilateral shift operator, and it is well known (see, for example, Theorem 2, [2]) that a bilateral shift operator always generates a v. N. algebra that is maximal abelian. It follows that $L$ must generate $R$, and since $\phi L \phi^{-1}$ is the bilateral shift operator $L^{\prime}: z^{n} \rightarrow z^{n+1}$ in $\widetilde{\boldsymbol{R}}, \phi \boldsymbol{R} \phi^{-1} \subset \widetilde{\boldsymbol{R}}$. But $\phi \boldsymbol{R} \phi^{-1}$ and $\widetilde{\boldsymbol{R}}$ are both maximal abelian v. N. algebras, and it follows easily that $\phi \boldsymbol{R}_{\phi}^{-1}=\widetilde{\boldsymbol{R}}$. This completes the argument.

5. Concluding remarks. We first point out that the definition given above for a Riesz system could be loosened somewhat without affecting the results of $\$ 3$. In particular, if the v. N. algebra attached to a Riesz system is required only to be abelian and have no minimal projections, then the proofs in $\$ 3$ carry over without change. Furthermore, many of the results of $\$ 3$ remain true if the definition of a Riesz system is weakened further by requiring only that $\mathscr{K}^{\perp}$ be a weak Riesz subspace for $\boldsymbol{R}$. In particular, Theorems 1,2 and 3 remain true in this setting.

One can also regard the results of $\S 3$ as theorems about a single operator that has a normal dilation with the appropriate properties to give rise to a Riesz system. The interested reader can work out the precise formulations of the results of $\$ 3$ from this standpoint for himself.

It appears to us that further inquiry into the properties of (GT) operators might well be fruitful. In particular, one would like to know if there is an analogue for analytic (GT) operators of the Beurling theory of invariant subspaces for analytic Toeplitz operators, and if the spectrum of 
every (GT) operator is necessarily connected. (The fact that the spectrum of every Toeplitz operator is connected was recently proved by Widom [11].)

\section{BiBLIOGRAPHY}

1. A. Brown, On a class of operators, Proc. Amer. Math. Soc. 4 (1953), 723-728.

2. A. Brown and P. R. Halmos, Algebraic properties of Toeplitz operators, J. Reine Angew. Math. 213 (1963), 89-102.

3. J. Dixmier, Les algebres d'opérateurs dans l'espace Hilbertien, Gauthier-Villars, Paris, 1957.

4. P. Hartman and A. Wintner, The spectra of Toeplitz's matrices, Amer. J. Math. 76 (1954), 867-882.

5. H. Helson and D. Lowdenslager, Prediction theory and Fourier series in several variables. II, Acta Math. 106 (1961), 175-213.

6. K. Hoffman, Banach spaces of analytic functions, Prentice-Hall, Englewood Cliffs, N. J., 1962.

7. F. Riesz and M. Riesz, Über die Randwerte einer analytischen Funktion, Quatrième Congrés des Mathématiciens Scandinaves, Stockholm, 1916; pp. 27-44.

8. I. E. Segal, Decompositions of operator algebras. I; II, Mem. Amer. Math. Soc. 9 (1951).

9. __ Equivalences of measure spaces, Amer. J. Math. 73 (1951), 275-313.

10. G. Silov, On the decomposition of a commutative normed ring into the direct sum of ideals, Mat. Sb. (N. S.) 74 (1953), 353-364. (Russian).

11. H. Widom, On the spectrum of a Toeplitz operator, Pacific J. Math. 14 (1964), 365-375.

UNIVERSITY OF MICHIGAN, ANn Arbor, Michigan 